\title{
The Optimization Study on Passenger Organization at Passenger Station Based on CAS
}

\author{
Wei Wang ${ }^{1}$ Li Huang ${ }^{2}$ \\ ${ }^{1}$ Transportation \& Logistics Engineering Institution, HoHai University, Nanjing 210098, P. R. China \\ ${ }^{2}$ PHD of Business School, HoHai University, Nanjing 210098, P. R. China
}

\begin{abstract}
The paper combines the passenger behavior with the decision of administrative department based on multi-agent simulation method of complex system theory, considering each advantage of CA and multi-agent, with the entrance of administrative departments and passenger behavior. In the passenger organization optimization model involving in different kinds of agents such as passenger organization agent and passenger agent, which combines multi-agent with $\mathrm{CA}$, the environment factor is expressed by CA and the paper constructs multi-agent behavior decision model depending on the basic frame of cellular automata (CA) pedestrian flow. Individuals respond to the changes of environment around through the interaction between resource and the environment or negotiating with another individual. Through simulating the complex process of decision making of administrative departments, it brings forward the technical route and the basic frame of the passenger organization optimization of large-scale passenger station on complex adaptive system(CAS), constructs the optimization system of passenger organization based on multi-agent, discusses the structure and the competing and cooperating relationship between each agent, and designs the optimization arithmetic based on genetic algorithm(GA) of multi-agent. At last, taking the optimization of passenger organization during rush hours at Guangzhou Station as the example to analysis and getting a good effect, this can offer the reference to the decision making of administrative departments.
\end{abstract}

Keywords: Complex adaptive system (CAS), Passenger organization, Cellular automata (CA), Multi-agent, Genetic algorithm (GA)

\section{Introduction}

The characteristics of passenger organization project are that, large pedestrian flow, great changes of pedestrian flow, great frequency, heavy strain of equipment ability, and complex of passenger organization, which need the unitary cooperation of each type of work and working procedure, need the comprehensive utilization of each equipment, and even more need the optimization and cooperation as a whole.

The study of the passenger organization optimization we have now is less, most of which is qualitative analysis. The quantity analysis can only be found in the study of YanYusong and Li Wenxin ect., in which, they bring the computer simulation technology into the project optimization of passenger organization at large-scale passenger station during rush hours [1]. Basing on the production of former study, considering the complicacy of passenger behavior in the simulation of pedestrian flow and the interaction between the passenger behavior and the environment around, the paper is adopting CA and multi-agent technology to simulate pedestrian flow effectively for better reacting the subjectivity and randomicity of pedestrian flow at station.

The use of CA model in transportation flow has been many years and has got a lot of achievements [2]. Because the simulation of pedestrian flow involves in behavior characteristics of people, there is a great advantage to study it with agent. Therefore, CA and multi-agent technology can deal with this problem better and simulating pedestrian flow effectively. Simultaneity, Ref. [1] adjusts the project by manual work according to simulizition effect and has got a good effect, but passenger station system is a typical opening complex system, the non-linear relationship of each factor and the coexistence of advantage and disadvantage power that lead to the fuzzy and uncertainty of the system development, which make it harder to optimizing passenger organization. The method emphasizing on qualitative study or quantitative simulation with adjusting by manual work has been false to meet the integrated and dynamic need of passenger organization at passenger station. Because of human behavior involved, the study of transportation organization is always complex, so it would be more advantage to study this with agent.

According to the requirement above, the paper constructs the method system of passenger organization optimization at passenger station on CAS with the aim of exploring the optimization of passenger organization 
project at passenger station; brings theories of CAS and GA based on multi-agent to assure the integrity and dynamic of passenger organization at passenger station and the convergence of model; Combining CA with multi-agent to simulating pedestrian flow, and developing optimization software of passenger organization at passenger station with high portability to make useful exploring of the harmonious and sustaining development of our passenger organization at passenger station, and the result has proved that there is a good foreground for this theory and method to be used.

\section{The technical route of passenger organization optimization at passenger station on CAS}

Considering the dynamic and non-linear characteristics of passenger organization at passenger station, the traditional way can't discribe and deal with the problems well. So, the paper simulates the real optimization process of passenger organization through divding the optimization problems into two phases: the first phase is optimization, and the second phase is the simulation of pedestrian flow project. During the optimization phase, a passenger organization project is given from the perspective of system optimization, then entering the simulation phase to simulate the project,

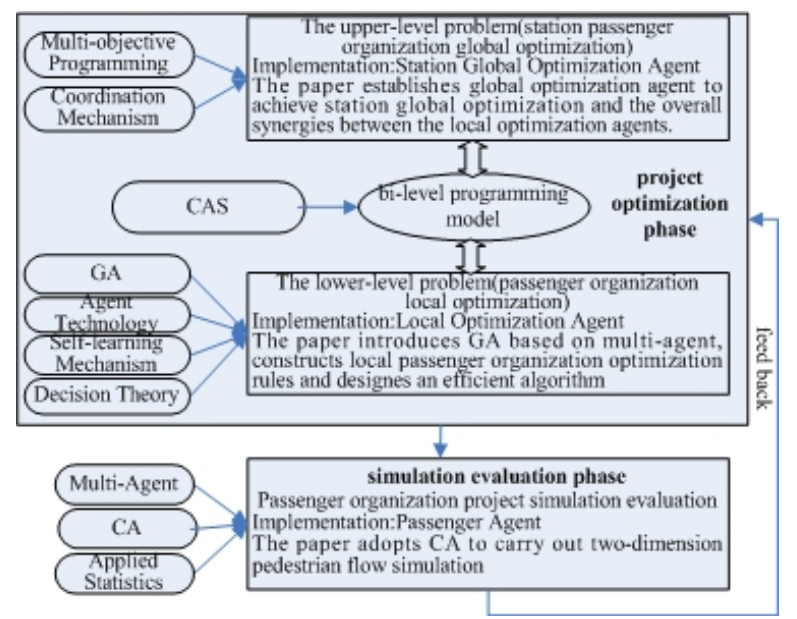

Fig. 1: The technical route adopted.

passengers choose their walk behavior by their own benefit and preference, the information of the pedestrian flow is feeded back to the optimization phase, and the project is adjusted according to the traffic flow distribution characteristics and the basic requirement of passenger organization, and then do the simulation again, in which the passengers choose again in the new project, and repeat that for many times, then the passenger organization optimization of project can be got. Constucting the bi-level programming model optimization model during the phase of optimization, the upper-level optimization is the station global optimization problem, and the lower-level optimization is the local optimization problem. The bi-level optimization model considers both the basic requirement of passenger station and the real need of transportation to adjust the project, designing the decision project together. The appraisement of passenger organization project is achived by pedestrian flow simulation, which can't change the project, but can reflect the choice of passenger behavior, and bring the transportation effect of the project back to the bi-level optimization model to adjust the project. The technical route is shown in Fig. 1.

\section{The construction of passenger organization optimization system at passenger station based on multi-agent}

\subsection{The system architecture framework}

Passenger organization optimization system at passenger station adopts multi-level hierarchical structure, and each level is composed of agents with the same function and structure, including Station Global Optimization Agent(SGOA), Local Optimization Agent(LOA) and Passenger Agent(PA) [3].The system framework is shown in Fig. 2.

SGOA optimizes system project from the perspective of passenger station, and brings in synergy mechanism to achieve coordination optimization between LOA; LOA answers for passenger organization project of each agent( including organization control points such as: waiting room, temporary waiting room, platform, passageway etc.) the optimization of bi-level optimization model is constituted by both SGOA and LOA. SGOA as the coordination unit of LOA makes sure the cooperation policy and reference between them; LOA should transfer the reactive information between local system environment and the system optimization capability to SGOA as the decision reference. The agents of LOA can cooperate themselves, and then enter into station global optimization if they are false. Just like that, each LOA is independent in some extent but not all, which is going to work when, receives the requirement of another LOA and through SGOA to achieve station global optimization and optimization of passenger organization. 
The passenger organization project produced by bi-level programming model optimization model leads the behavior of PA, carries optimization of pedestrian flow, and feeds back the optimization result to the bi-level optimization model. According to the optimization result, the model makes further adjust of the project.

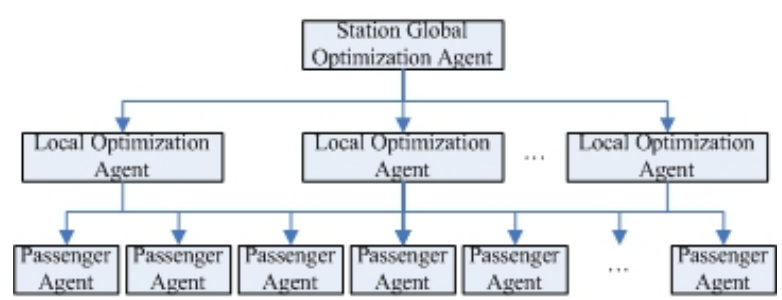

Fig. 2: The system framework of passenger organization optimization at passenger station.

\subsection{The internal structure of LOA}

Each local region decides optimization project according to its own situation for adapting to dynamic change of local pedestrian flow, which is local optimization problem. LOA is the basic and primary part of optimization system, which decides the passenger organization project according to its aim, capacity, knowledge and the data obtained. The inner structure of LOA includes: (1)Rules base. Storing the arriving rule of pedestrian flow, local correlative information, the information and pedestrian flow rule of nearby region; (2) Fusion algorithm module. Carry fusion disposal to the forecasting parameters of basic transportation(such as flux, speed and occupancy rate), and then get the suitable parameters of basic transportation; (3)Knowledge base. Store the history information of passenger organization optimization of LOA. The information include time, pedestrian flow information, optimization project and its effect etc., which is a individual knowledge gather about local passenger organization optimization; (4) Learner. Learner is a gather of several methods and rules; (5) Coordination module. Manage and control the information transferring between agents, and produce optimization project through interacting with Knowledge module. The inner structure of LOA is shown in Fig. 3.

Passenger Flow Simulator optimizes the passenger flow according to the forecasting data, which sends the simulation result of passenger flow to optimization module for primary disposing. After eliminating unsuitable data and supplementing absent data, the optimization module passes the data to fusion algorithm module for fusion calculating, and then the suitable parameters of basic transportation can be obtained. Learner interacts with knowledge base and rules base unremittingly, according to relative information received and experience or quantity information obtained by learning, and adjusts learning according to the true situation of LOA. The close LOA and SGOA act on knowledge base and optimization project through the function of coordination module, and organize the behavior of PA. Information fusion technology is pivotal in the forming process of optimization project. Agent carries fast fusion disposal of the information from simulator and different channels with the basis of information fusion module to form useful and correct data and share information between agents, which is good for the cooperation between agents and the whole system optimization.

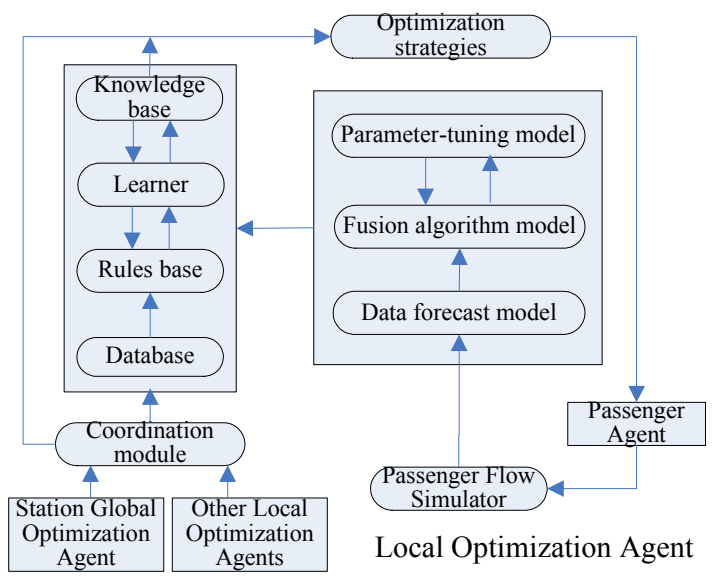

Fig. 3: The inner structure of LOA.

\subsection{The internal structure of SGOA}

SGOA aims to achieve the knowledge share and cooperation between LOA, which adopts cooperation way to disassemble and synthesize assignments, according to the information received from each LOA and the operation way of decision(knowledge share or cooperation) for achieving the cooperation control of searching space and evolutionary parameter of each LOA, avoiding the possible result conflict, and achieving information management of all cooperation information and information interaction with LOA.

The structure of SGOA is nearly the same as LOA, however, the difference between them is that, SGOA has self-contained coordination unit besides global program base, fusion algorithm module, experience base and learner, see to Fig.4. Coordination unit achieves knowledge share and cooperation between lots of LOA when the capacity of LOA can't meet the assignment need. According to the assignment need of LOA, SGOA decides the cooperation way through 
judging the matching rate between itself and the assignment need. If it meets the need of assignment with limit knowledge, it will cooperate with another LOA for knowledge share; if it is false to deal with problems by itself, it will adopt the way to disassemble assignment cooperating with another LOA, and control the representative individual in coordination process.

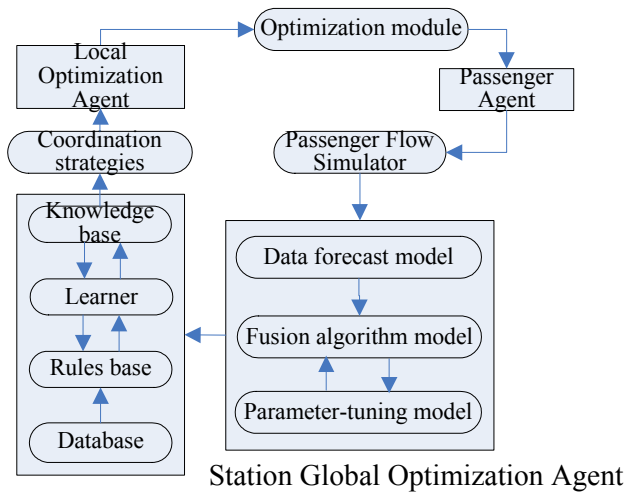

Fig. 4: The inner structure of SGOA.

\subsection{The internal structure of PA}

PA is an agent abstracted from passenger working behavior in real transportation, which can get information from outside automatically, and adapt its working behavior to situation. The model structure of PA is shown in Fig. 5. PA not only keeps reacting to the changes outside, but also has complex judgment capacity and its own knowledge to adjust its behavior according to the transportation situation outside.

The basic framework of model is dividing the station plane equally, and using agent to show the pedestrian, whose behavior follows the local rules and adapts to environment change. Each cellular of the model corresponding to space $0.40 \times 0.40 \mathrm{~m}^{2}$, which is the typical space distribution of people in a dense pedestrian flow. All of people in the model change their place synchronously, so the reactive time is decided both by cellular space and pedestrian rate because of time problem. In the real crowd movement, people have strong independent ability, they make corresponding judgments and decisions to the environment around. The main behavior of agents in the model is to make sensible choice for next step according to the environment around they have known. Each agent has a visual field and only feels the situation in some range, so we choose Von Neumann area to show the visual field of each agent in the model, and the behavior of each agent is totally up to the environment of its visual field. We adopt a simple hypothesis in the model like that, if there would be more than 2 people to compete at the same point after primary judgment of agents, who may give up the competition to choose another sub one. Here we will describe a piece of attractive level map for each agent, which is the distribution map to show in what extent each cellular attracts its own agent. In the simulation process, supposing the probability of each agent to choose a cellular around as its movement way is direct to the attractive value of the cellular. What need to be pointed out is, each agent can adjust the cellular attractive level map as time changing, so the change of attraction is to some extent controlled by passenger organization project [4].

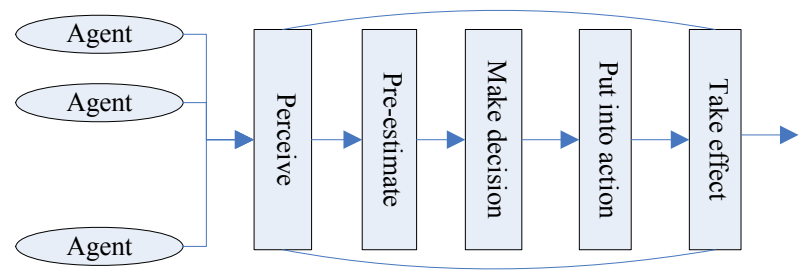

Fig. 5: The structure and function of PA.

Bring in individual fitness to describe the influence rate after individual choice of different strategies. The PA project includes route choice project and the stop point project of agent. The individual behavior project of passenger is shown by the transportation effect of passenger organization project through transportation simulation, and react that to SGOA and LOA as the basis for project adapting.

\section{Optimization algorithm}

\subsection{The main thought of optimization}

Bi-level programming model is NP-complete problem, and genetic algorithm (GA) is a better solution. We use GA in project optimization, and combine CA with multi-agent technology to have the passenger flow simulation evaluation. Optimization module is composed of two main components: GA Optimizer and Passenger Flow Simulator. GA Optimizer produces the initial individuals, every individual is evaluated by Passenger Flow Simulator and gains the fitness value, and the next generation individuals are gained evolutionarily by GA Optimizer according to the fitness values. So the cycle is going on until the termination conditions are met, the framework is shown in Fig. 6 [5]. 


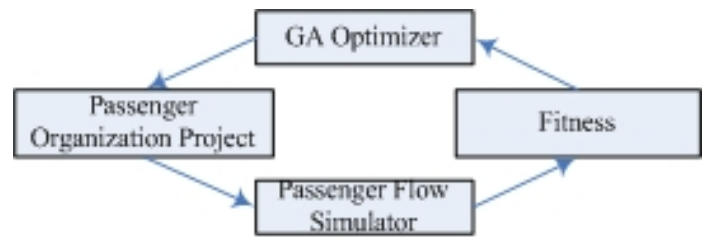

Fig. 6: The total framework of passenger organization optimization algorithm.

\subsection{Optimization algorithm}

To assure the integrity and dynamic of POOS, We introduce GA based on multi-agent to settle the bi-level programming model of the passenger organization optimization. We analyze fitness function of algorithm, disposal of restriction conditions and design of genetic operators concretely below. In optimization model of passenger organization at station, global optimization is achieved by SGOA. Meanwhile, SGOA is also responsible for the synergy optimization between LOA; local optimization is realized by LOA.

1) Fitness Function

The fitness function of the algorithm adopts the objective function or the evaluation function of the optimization project. The fitness function is shown in Eq. (1) below.

$$
F(i)=A_{\max }-A(i) M / \sum A(i)
$$

In Eq. (1): $A_{\max }$ is the max. of $A(i) M / \sum A(i) ; A(i)$ is the objective function of the individual $\mathrm{i} ; \mathrm{M}$ is the number of LOA; Moreover, when local passenger flow exceed the resource limit or local queue length exceed the queue length limit, we reduce the penalty factors from the fitness function for treatment.

2) Disposal of Restriction Conditions and Design of Genetic Operators

Because agent is more complicated than coding string, the genetic operators in GA based on multi-agent are more flexible than SGA, and the kinds of operators are more [6]. The paper adopts the genetic operators as follows:

(1) Selection Operator: Selection Operator chooses the roulette election operator based on "arrangement place".

(2) Local Adjustment Operator: Adjusting the passenger organization project of local agent dynamically according to passenger flow and resource limit of each agent, the formula is following:

$$
S^{t}=f\left(\Omega, S^{t-1}, \phi(N), A, R^{t}\right)
$$

In which, (1) $\Omega$ - shows the place of LOA, here $\Omega=\{\mathrm{i}=1, \ldots, \mathrm{N}\}$; (2) $S^{t}$ and $S^{t-1}$ - show the projects of LOA at time and $\mathrm{t}-1$ time; $(3) \phi(N)$ - shows the region of LOA for interaction; (4) $R^{t}-$ shows the fitness of
LOA at $\mathrm{t}$ time, which show the influence rate to benefit after different projects choused by local agent ; (5) $f$-shows the rule function of situation transformation.

(3) Coordination Operator: Coordination operator is used to achieve the coordinative optimization of LOA. In the optimization system of passenger organization, a decision made by an agent always influences other' $s$ decision, and is influenced by other's simultaneously. The passenger organization optimization of a single local agent has to consider the situation of other local agents. If the local agent can't settle the occurent problem, it must ask another nearby agent for help to achieve global optimization through the coordination between LOA. In the optimization process of passenger organization, the distribution of passenger flow is carried one after one with sequence, based on which, the paper adopts one-step Nash equilibrium to achieve the coordination between agents and the global optimization [7].

Because game theory studies the problem of individual choice with external restriction, the use function of the individuals not only relies on self-choice, but also depends on other's choice. Considering the instability of the resource and information belonging to each agent and the increasing of the dimension of agent and passenger train lines, the count become more difficult, base on which, the distributing of passenger flow is working with vehicle order as a unit, in the model of passenger organization project, so the paper will deal with that problem with one-step Nash equilibrium, considering the best way of agent, we make that

$$
V_{i}\left(S_{i}^{*}, S^{*}{ }_{-i}\right) \geq V_{i}\left(S_{i}, S^{*}{ }_{-i}^{\prime}\right), \quad \forall S_{i} \in \sum_{i}
$$

In which, $S^{*}{ }_{-i}$ is the best unite way of other individuals apart from ' $i$ ' in previous step, which is the best way of one-step.

(4) amendatory operator: The penalty function would be used when regional passenger flow exceeds the restriction of resource or regional queue exceed the restrictive length.

The paper adopts 4 genetic operators, which are choosing, local adjustment, coordinating and amending, adopts roulette selection operator and the best way of one-step to coordinate. The 3 operators above may make unsuitable project, so it is needed to construct an amendatory operator similar to aberrance operator to coordinate. The main function of the amendatory operator is checking the result after the 3 operator above according to the restriction, if uniformity, imperative aberrance with probability would be used to coordinate till last according to the fitness function of the penalty factor added into coding. 


\subsection{Calculating process}

The calculating process of passenger organization optimization algorithm is shown in Fig.7.

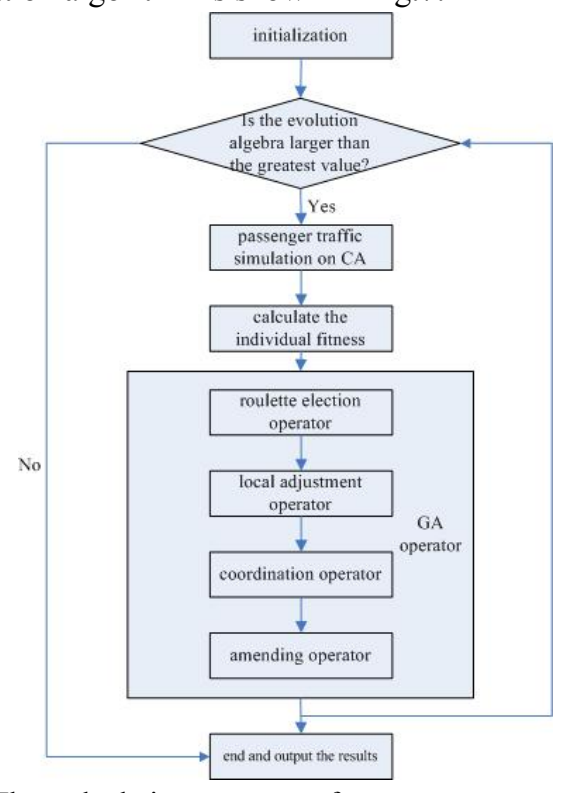

Fig. 7: The calculating process of passenger organization optimization algorithm.

\section{Empirical analysis}

The paper takes the passenger organization optimization at Guangzhou Station during rush hours of Spring Festival in 2003 for example. Therefore, the paper set up the simulation platform, in which passenger flow simulation adopts cellular automata model. Moreover, passenger flow is simplified into arrival flow and departure flow according to the objective of the passengers at station. Passenger flow lines are shown in Fig.8, Fig.9 and Fig.10.
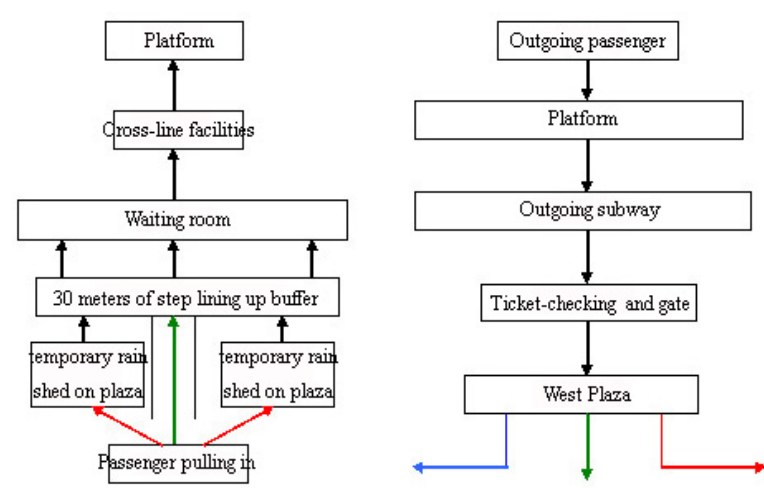

Fig. 8: Passenger pulling in and outgoing flow lines at Guangzhou Station during rush hours.
The regional scenes in simulation are consistent with the actual scenes at station completely. There are seven ways for entering and four ways for going out at station. The arrival laws of passengers are gained by the questionnaire survey of the passengers, the interviews with the station staffs and the actual estimations at site. The service time at each operational site adopts the actual measured data and the passenger flow speed between regions can be computed by the distance between the regional divided by the average consumptions. Based on the investigation and the experience value, the peak utilization area per capita of the waiting room adopts $0.5 \mathrm{~m}^{2} /$ people at Guangzhou station. Because of without chairs in the temporary waiting rooms, the peak utilization area per capita adopts $0.4-0.6 \mathrm{~m}^{2} /$ people.

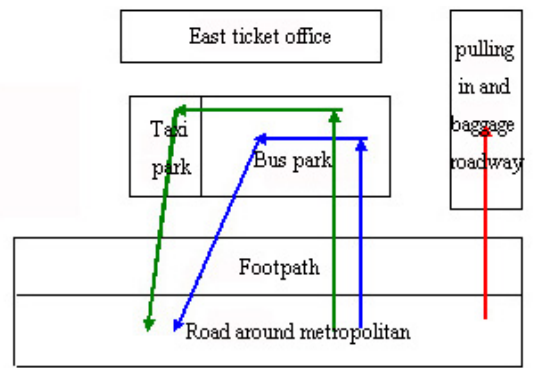

Fig. 9: Passenger booking flow lines at Guangzhou Station.

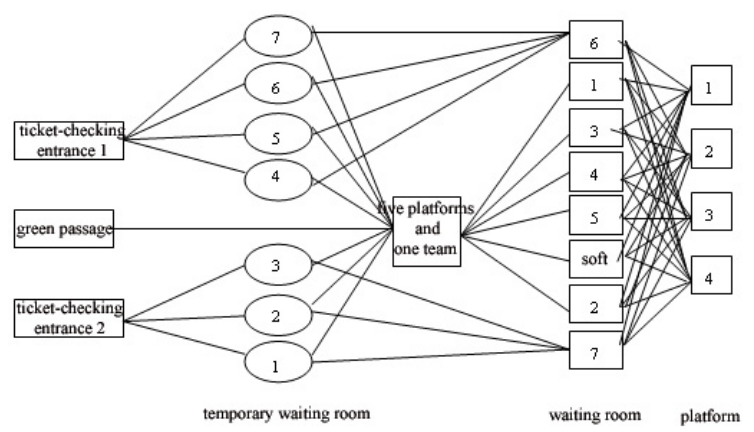

Fig. 10: Passenger flow lines sketch map at Guangzhou Station.

The biggest evolutionary algebra of GA is 100 , crossover probability is 0.9 and mutation probability is 0.01 . Here optimization objectives are the maximum of the overall coordination of resources and minimum of the total walking time of all passengers. And the evolution ends at 100 algebra, then a satisfactory solution is gained. The curves on the evaluation scores of passenger organization project at the process of simulation are shown in Fig. 11. Fig. 11 shows that after 100 times evolution the passenger organization project is optimized greatly, the result is reasonable and agreed with expectation. 


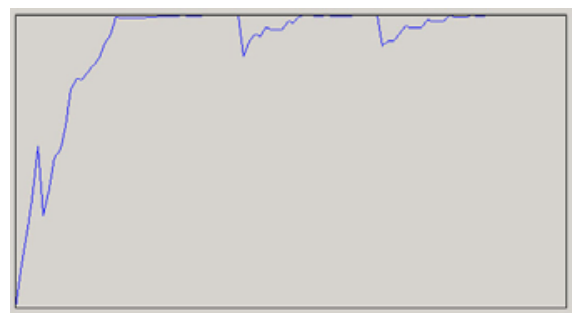

Fig. 11: The curves on the evaluation scores of passenger organization project at the process of simulation.

\section{Conclusions}

The paper brings forward the study way of the passenger organization optimization at large-scale passenger station during rush hours on CAS, constructs bi-level programming model and finds the answer of it through GA based on multi-agent, which is used in the optimization of passenger organization at Guangzhou Station during rush hours and gets a good effect, offering a reference way for the further study of passenger station, freight-transport station and the system optimization of logistics park. Because of the restriction of study ability and time, the achievement of this paper is preliminary only, and there are some shortages in the paper, but we will solve these problems in future demonstration investigation and study.

\section{References}

[1] Y.S. Yan, W.X. Li, Optimization Simulation of Passenger Organization at Passenger Station during Passenger-flow Rush Hours. Computer Simulation, 21: 167-170, 2004.

[2] V.J. Blue. J.L. Adler, Cellular automata micro simulation for modeling bi-directional pedestrian walkways. Forthcoming in Transportation Research B-METH, 35: 293-312, 2001.

[3] J.Y. Zhao, L.P. Zhao, Urban traffic flow control prototype system based on multi-agent. Journal of Traffic and Transportation Engineering, 3: 101-105, 2003.

[4] W.F. Fang, L.Z. Yang, R. Huang, A Multi-agent Model Based on Cellular Automata for Simulating Human Behavior to Assess Building Design Performance. Engineering Science, 5: 67-71, 2003.

[5] R.M. Li, W. Li, The Integrative Modeling of Dynamic Traffic Assignment and Traffic Control in Saturated Networks. Information and Control, 33: 641-645, 2004.

[6] H.K. Zeng, D.Y. Sheng, Study of a new multi-agent based genetic algorithm and its application in complex system. Information and Control, 32: 277-280, 288, 2003.

[7] G.Q. Wang, J.Q. Xu, H.B. Yu, A Multi-agent System Task Competition Model and Algorithm Research. Information and Control, 34: 434-438, 2005. 